\title{
Realistic Modeling of Agents in Crowd Simulations
}

\author{
Mirza Waqar Baig ${ }^{1,2}$, Emilia Barakova ${ }^{1}$, Carlo S. Regazzoni ${ }^{2}$ and Matthias Rauterberg ${ }^{1}$ \\ Department of Industrial Design Eindhoven University of Technology, Eindhoven, Netherlands ${ }^{1}$ \\ Department of Biophysical and Electronic Engineering, University of Genoa, Italy ${ }^{2}$ \\ \{M.W.Baig, E.I.Barakova, G.W.M.Rauterberg\} @ @tue.nl, Carlo.regazzoni@unige.it ${ }^{2}$
}

\begin{abstract}
Simulation of crowd behaviors is a widely discussed topic for over a decade for computer games, architecture and entertainment. The most important thing is that agents should execute the human like behaviors in real life scenarios. This situation becomes very complex with dense crowds. Our proposed Realistic Behavior Agent Simulation (RBAS) model simulates the agents from the knowledge of empirical findings of crowd physical behaviors and crowd psychology. This paper presents a novel technique to design the agents to with following modules 1) Path planning behavior for collision avoidance 2) Situation awareness during herding behavior and turbulent flow in high density crowds. 3) Personal Reaction bubble (PRB) based response and perceptions. The evaluation with real life situations is performed to validate the RBAS model. The RBAS model allows the agents to use cognitive understandings to plan ahead their path using the visual perception information. This model encapsulates the emergent crowd behaviors such as self-organization behavior in herding situation. During turbulent high density flows, most of the existing models fail to predict the behavior of agents, the evaluations show the RBAS model mimics the same behavior of the crowd in different situations.
\end{abstract}

Keywords- Crowd Simulation, Agents modeling, Collision Avoidance, Situation assessment

\section{INTRODUCTION}

When a large number of people gather in a confined space or area, they make a crowd. It is difficult to know that how they will move and pass through a confined space and how the buildings and pathways should be built such that people passing through them do not have situation of congestion and jamming and they pass easily without injuries or problems. It is cumbersome and dangerous to bring a large number of people along and to perform the experiments, therefore it is feasible to make the realistic simulation and making the model and then collecting real users data based on the results from that model.

The crowd of people is a complex design system. To handle the complexity, one way is to model the situation comprised of individual people into simulations and making the results based on them. Such techniques are called agent based modeling and are related to social sciences areas and introduce randomness in model. They are already in use in different and diverse areas, such as molecular biology, economics and physics. Human behaviors are complex emergent phenomena, which are difficult to encompass using just mathematical or physics knowledge. The basic steps to make simulations are 1) Designing of agents with sufficient information which should enable them to behave same as pedestrians in simulation 2) Creating different kinds of real life situations such as panic situation and evaluating the behavior of agents, which should be same as real life. There are different crowd simulation models which can be divided into three types 1) Cellular automata 2) Velocity based 3) Flow based. Cellular Automata based discrete models have been in use mostly for architectural purposes [1][2]. These models define the two dimensional space with fixed blocks and occupants can move from one block to another following the movement rules such as keeping in view the free space if not occupied by another agent or obstacle. These type of models failed to simulate the situations when high density crowd is encountered as agents get limited only to restricted moments, therefore during turbulent flow situation, it fails to portray well these situations. Later, [3] the game based models have introduced the plenty based payoff rules to improve the collision avoidance rules. But still it lacks in mimicking the turbulent flows in high density crowds efficiently. Velocity based models emerged from research in game designs. [4][5] design the agents to select their behavior mostly from choosing the direct path to the destinations and changing their velocities. These models design the agents to plan their paths ahead to avoid collisions but they also lack in planning the variable velocities in high density crowds as every condition changes during high density crowds due to turbulent flows. In flow based models, the most famous is social force model [6], which have been used with different modifications [7][8]. This model models the agents as particles which move under effect of different kinds of forces. The proposed RBAS model is based on social force model and defines particles as agents which have capability to plan their paths to avoid collisions. During the turbulent flows, the agents behave as sliding bodies which gives the exact situation of a real crowd.

\section{PROSPOSED MODEL}

The proposed model introduces the realistic crowd behaviors based on cognitive and physiological findings and have been demonstrated in path planning, situation awareness. To design the realistic simulations the physical size of the agents is very important, it should be same as real life pedestrians. The physical size is important to simulate turbulent flows and egress analysis [9]. The agents in the simulator have been designed as ellipses with derived dimensions from Architectural graphic standards [10]. The model is based on Helbing model [6]. The agents in our model have same forces as SFM [7]. Borrowing the concepts 
from physics, the social forces concept means that forces are being modeled based on laws of motion such that person movement is due to the resultant forces acting on it, which in result will produce acceleration and thus affect the movement of the person. The resultant force on an agent $\mathbf{a}$ is given by, $\vec{f}_{r}$ is the sum of three main forces and one random force $\vec{f}_{\text {ran }}$;

$$
\begin{aligned}
& \vec{f}_{r}(t)=\vec{f}_{a m}(t)+\sum_{a \neq b} \vec{f}_{a b}(t)+\sum \vec{f}_{a w}(t)+ \\
& \vec{f}_{\text {ran }}(t)+\vec{f}_{\text {res }}(t)
\end{aligned}
$$$$
\text { where desired motivation force } \overrightarrow{f_{a}^{m}} \text {, the interaction force }
$$

$\vec{f}_{a b}$, the repulsive force $\vec{f}_{a w}$, the resistive force $\vec{f}_{r e s}$, the random force $\vec{f}_{\text {ran }}$ is used to induce noise and random behavior in subsequent simulations to produce multiple unique behaviors. In [11] Mehran et al. explained the abnormal behavior in real life scenes using social force model. But he used the social force model without any randomness factor in social force and explained that he detected dynamic crowd behavior in real life scenes using social force model without randomness in model. We call it this randomness force as random behavior and to include this randomness in simulator, we used the random force $\vec{f}_{\text {ran }}$ in resultant force $\vec{f}_{r}$. This also tends to make random situations and unpredictability which are the key features of real life scenarios. The particles in the crowd simulator have been included with a random force, which is based on the Gaussian distribution of the previous force of the particle. The random force $\vec{f}_{\text {ran }}(t)$ is generated by the Gaussian normal distribution with $\mathcal{N}\left(0, \frac{1}{k} \vec{f}_{r}(t-1)\right)$, where mean is 0 and variance is $\left.\frac{1}{k} \vec{f}_{r}(t-1)\right)$ in which $\mathrm{k}$ is the scaling factor and $\left.\vec{f}_{r}(t-1)\right)$ is the resultant force on particle in previous time instant. The forces $\vec{f}_{a b}$ and $\vec{f}_{a w}$ change themselves during turbulent flows in high density situations as explained in section B.

\section{A. Path planning for collision avoidance}

The SFM model based of flow of gas molecules has drawbacks that agents do not have information of the environment. Therefore, during the simulations the agents try to engage in situations earlier than expected and undergo rigorous collision due to effect of immense forces in high density crowds. The particles in SFM model modelled on physics based rules try to create panic earlier than expected. The agents in RBAS model plan ahead to avoid collisions and choose the path with minimum effort and collisions. The path planning module is based on the fact the when pedestrians walk in any area, they look around and plan their trajectories by estimating the movements of other pedestrians around and also taking into effect the interaction effects from them. This area is called information processing space IPS [12]. Using head mounted eye tracker and laser range scanners in controlled environment, the IPS came up with important observations. The pedestrians pay more attention to the objects which are in front at smaller distances than side objects. The pedestrians pay more attention to people in field of vision (FOV) with $90^{\circ}$. The static obstacles are more important than moving obstacles. Using these findings, a fan shaped IPS is proposed for each agent. The IPS changes its range based on speed variations. It goes to $180^{\circ}$ during turbulent flows. During normal walking, the IPS has sectional angle of $90^{\circ}$. The IPS is a spatial area in which agent finds possible interactions with other agents or wall. IPS around an agent is shown is figure 1.

The collision estimation in crowd simulation is based on detection of people moving with variable speeds and their impact estimation on agents. In previous studies such as [13], a collision prediction angle based on other person direction and gaze direction angle was used to identify the collisions. Most of the algorithms focus on collisions only, but they do not care about the collision types.

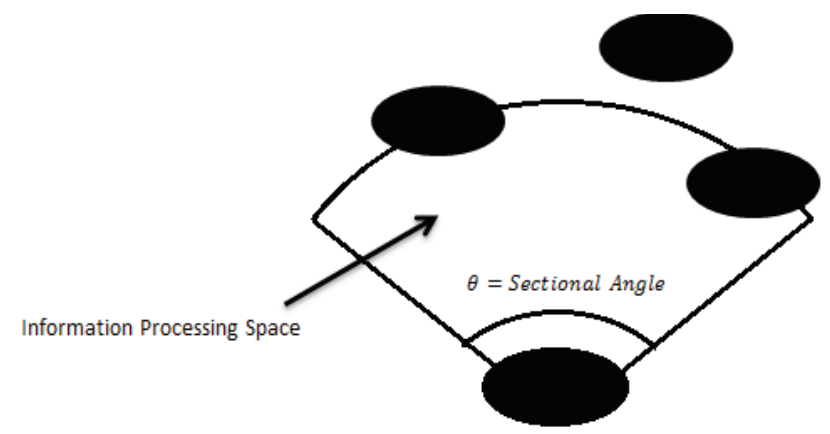

Figure 1. IPS around an agent in proposed model

The collision types are of much importance to plan the path and to interact with other agents and environment. The collisions can be of three general types i.e., (i) Head on collision (ii) rear end collision (iii) side collisions. These collisions are estimated by the agents for planning path ahead. From psychology [14], the agents take the paths with priority where they have no chance of collisions or if there is a chance then it should be side collisions. Therefore, the type of collision estimation is important. In real life, the pedestrians estimate their path and try to avoid head on or rear end collisions and there are chances of side collisions. To embed this quality in agents, a collision control function counters for the estimation of all the three kinds of collisions. The agent detects the expected interaction response in one simulation cycle and changes its direction again in next simulation cycle based on estimation of next interaction. This cycle performs as cognitive cycle. The function used to estimate collisions between the agent $\mathrm{A}$ and the agent under impact $\mathrm{B}$ is given by impact angle estimation $\theta_{n}$ and impact distance estimation by $I_{n}$ at simulation time $t$.

$$
\begin{aligned}
& \theta_{n}=\left(\theta_{d}\right)_{t+1}-\left(\theta_{d}\right)_{t} \\
& \left(\theta_{d}\right)_{t}=\left(\left|\varphi-\left(\theta_{A}+\theta_{B}\right)\right|\right)_{t} \\
& I_{n}=\left(\left(d_{A B}\right)_{t+1}-\left(d_{A B}\right)_{t}\right) \\
& \text { if } \theta_{n}>\cos ^{-1} \frac{2 r}{d_{t h}} \\
& \text { and } I_{n}>\gamma_{t h}
\end{aligned}
$$


where $\theta_{n}$ is the impact angle estimation and $d_{A B}$ is the calculated distance between agents $\mathrm{A}$ and $\mathrm{B}$ at simulation times $\mathrm{t}$ and $\mathrm{t}+1 . r$ is the radius of agent which is its semi minor axis of ellipse and $d_{t h}$ is the threshold distance between two bodies, which depends on situations during simulations and changes during normal and panic behaviors,. The $d_{t h}$ decreases in case of panic behavior and increases in case of normal behavior. It is normally equals to the personal area (as mentioned in Section C), during normal situations and in case of herding behavior and turbulent flows, it decreases to intimate area. $\gamma_{t h}$ is the threshold distance between the two agents and it is equal to semi major axis of intimate area. The eq. 4 and eq. 5 makes the agent to plan ahead its trajectory. For planning the path without collisions, the eq. 5 and eq. 6 should be true. The type of collisions depend on the $\theta_{n}$ and $I_{n}$, there will be head on collision, if $\theta_{n}$ decreases and distance $I_{n}$ also decreases

$$
I_{n}<\gamma_{t h} \text { and } \theta_{n} \cong 0
$$

The collision will be side collisions as far as $\theta_{n}>0$, and $I_{n}$ decreasing.

$I_{n}>\gamma_{t h}$ and $\theta_{n}>0$

The agent tries to avoid from head on and rear side collisions and if there is no other way, then there is chance of side collisions, which occurs mostly during turbulent flows. This impact prediction technique is quite useful to mimic the real life pedestrians. The impact estimation brings the sense of environment and interactions planning in agents. The agents do not act like gas particles which just diffuse and create a clogging at doorways and pathways.

\section{B. Situation Awareness}

The crowd simulation models currently in use have been designed for different situations, but a few models take into account the high density crowd situations, in which turbulent flows occur. The physical interactions among bodies change, causing unintentional movements that are not possible in real life heuristics. The agents keep pushing behavior and there is shaking effect during high density of agents, as agents designed on basic social forces keep on pushing and repelling other agents. In real life, during high density crowd situation, pedestrians usually produce sliding motion in crowds rather than just repelling interaction force. This change in SFM also produces the herding behavior, in which people give up their already defined directions and follow other pedestrians to get out of situation. A physical contact force is included in SFM model. This force is inspired by the contact force modeled by Moussaid et al., [15]. The force is given by body force and physical contact force, which counters for body and sliding movement during high density clogging. The revised inter-agent force is given between two agents $\mathrm{A}$ and $\mathrm{B}$ as:

$\vec{f}_{A B}=\left\{A_{A} e^{\frac{r_{A B}-d_{A B}}{B_{A}}}+k g\left(r_{A B}-d_{A B}\right) \vec{n}_{A B}+k g\left(r_{A B}-\right.\right.$
$\left.\left.d_{A B}\right) \Delta v_{A B} \vec{t}_{A B}\right\}$

In eq. 8 , the $k g\left(r_{A B}-d_{A B}\right) \vec{n}_{A B}$ part accounts for the body force and counteracts with the body compression, where $\mathrm{k}$ is a constant, $g(x)=0$ if $\mathrm{A}$ and $\mathrm{B}$ do not touch each other else it is equal to $x$. The $k g\left(r_{A B}-d_{A B}\right) \Delta v_{A B} \vec{t}_{A B}$ counters for sliding friction force during high density situations, able to produce turbulent flows and tangential motion in case of high density crowds. The body $\mathrm{B}$ is replaced with $\mathrm{w}$ in case of walls. The crowd under these forces act like sliding motion as occurs during high density crowd movements. One example of this is during Hajj pilgrimage [16].

\section{Personal Reaction Bubble}

The areas of attention and areas of perceptions of persons are defined by personal reaction bubble (PRB). These spaces define the areas of interaction of pedestrians and vary from culture to culture. This PRB is useful in defining areas of interactions of agents. According to Hall et al., [17], this personal reaction bubble consists of four layers model provides a framework to produce realistic simulations of the crowd. As the radii of circles vary from culture to culture, people have different perceptions depending on the kind of layer. Previously these layers have been designed either as circle shaped [18] or ellipse shaped [19]. We define these shapes as semi ellipses. From cognitive and psychological heuristics, these semi ellipse shape have proven to be true estimation of interaction areas. Only first area, intimate layer space is full ellipse, this is due to the reason that pedestrians feel rear end interaction forces only when they have other pedestrians in that layer. The other layers are semi ellipses as shown in figure. 2

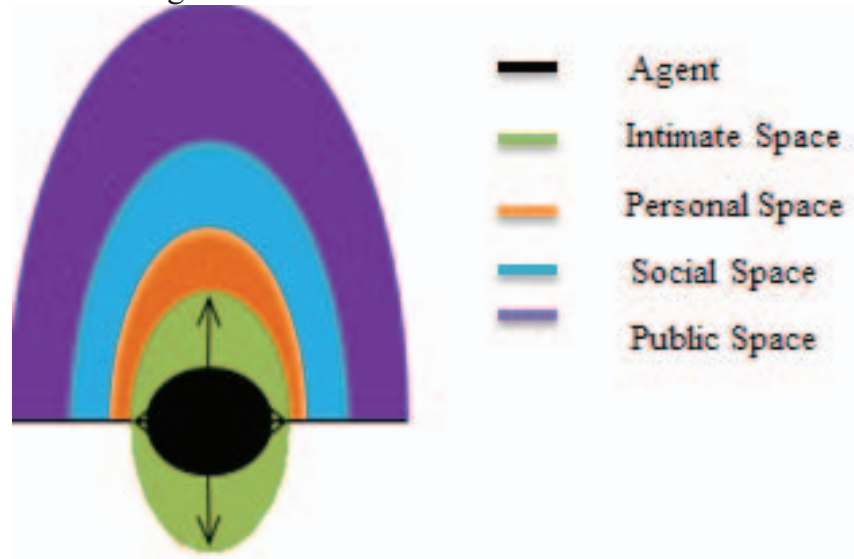

Figure 2. Personal Reaction Bubble for RBAS model. The four layers define the interaction layers of an agent.

\section{EXPERIMENTS AND RESULTS}

The crowd simulation model has been developed on $\mathrm{C}++$. The simulation model allows to create different scenarios and range of agents. The variable numbers of agents enter into the simulator at random positions and time. The agents have random birth rate (time to come in simulation) which is modeled by the poisson distribution. The time at which the particles enter is also randomly timed based on normal distribution. Figure 3. shows the crowd simulator 


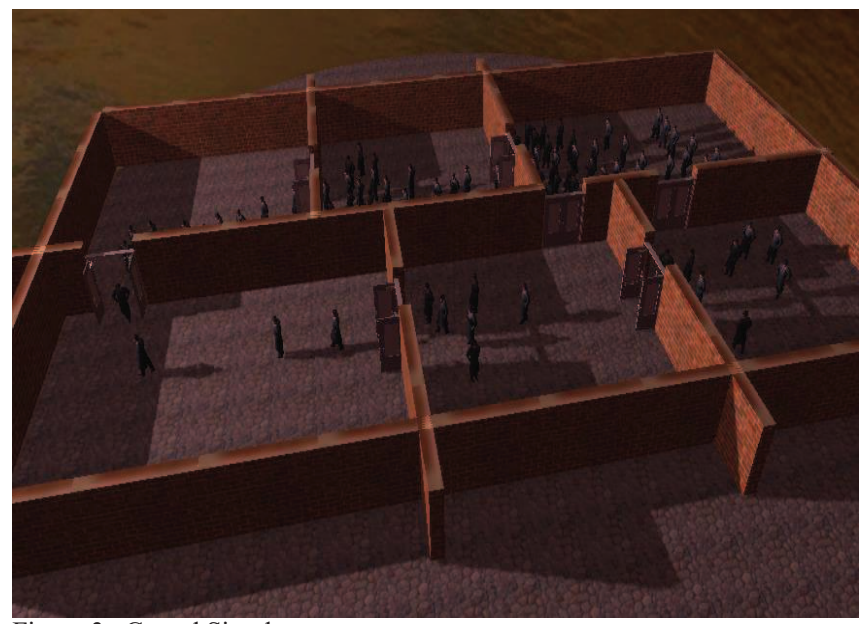

Figure 3. Crowd Simulator

The proposed model has been tested with different kinds of scenarios with varying densities. The model has been evaluated with SFM model and changes in model have been demonstrated. The RBAS model performance has been studied in following cases. The trajectories executed by the agents with the variations in density of people in simulation are performed. The ratio of trajectories executed to original trajectory versus density (agents/inch ${ }^{2}$ ) is plotted. Different types of flow of agents during simulations were tested. The original trajectory is the path taken by the agent during normal conditions i.e., laminar flow, the agent passes through the simulated environment without many interactions, this behavior is also observed during line formation situation especially at train, bus stations and narrow alley, as even the density increases, the crowd acts as self-organized to let the flow the agents. The stop-and-go waves event also appears, the ratio goes upto $110 \%$. The effort of agents to reach the destination increases as the density increases. This effect is also termed as faster-isslower effect [20]. During turbulent flows, the density increases from start with density, as the people are moving with much varying speeds in crowd. This turbulent flow describes the panic behavior in which agents mostly do collision avoidance more than situation awareness. During herding behavior, the effort to reach the destination increases, as the agents act like a group and follow the front agents. The agents access the situation and give up their primary path planning and do sliding motion among crowd and follow the other agents which are dependent on the flow of crowd also. This also demonstrates the group behavior. The effort to reach the destination in Herding behavior is less than turbulent flow. These results have been demonstrated in the Figure 4(a). The simulations have been performed with varying number of agents and simulations have been performed iteratively with varying parameters to validate the results. The density goes from 10 agents to 150 agents. The results have been shown in Figure 4 (a).

To evaluate our simulation model with real data, we performed the simulation by mimicking the exact scene from the videos of ETH walking pedestrian dataset BIWI [21], in which we collected the empirical data of spread of pedestrians in the street. The speed and occupancy of agents in street for 480 seconds is recorded. The same scenario was designed in simulation and first the SFM [6] based particles were and then our model RBAS was used. During these simulations, the data is collected using different flows such as unidirectional, counter, cross flow and all directional flows. The occupancy is defined as the area covered by the agents in case of simulation and by pedestrians in cases of ETH video. The agents vary from 0 to 30 and it corresponds to same number of people as in video. The trajectories have been plotted and demonstrated to show speed density relationship in Figure 4 (b). The results show that our RBAS model has the similar behavior as dataset, while particles in SFM model lacks to perform according to the situation.

The love parade disaster videos which were recorded just before the disaster have been evaluated to create the same environment in simulation. The pressure cue is used as feature to relate the same effect of behavior of agents with our model. The crowd pressure [15] is defined as local density versus local velocity variance. The calculations are done for 1000 frames of video right before the disaster where the turbulence started. The results have been demonstrated in Figure 4 (c). The results show that RBAS model creates the same pressure on given density as in the dataset.

\section{CONCLUSIONS}

This paper presents a novel approach to produce realistic crowd behaviors and RBAS crowd simulation behavior model is proposed. The algorithm simulates the agents as ellipse with particular sizes. The agents have sense of environment and plan their own path to avoid collisions. The shaking and repelling effect in agents have been reduced using the body contact and sliding forces. The interactions among agents have been modeled based on personal reaction space. This modification in SFM model makes the model more realistic as every parameter has been modeled from the results of phycology and video tracking.

Finally, the RBAS model has been tested for different types of crowd situations such as laminar, stop-and-go wave, turbulence and herding behaviors. The evaluations of the model are performed as compared with SFM and empirical data. The love parade disaster event was also evaluated using our model. The results show that our RBAS model outperforms in diverse situation and models the realistic agents in crowd. This makes this model to overcome the problems of models already in use. As evaluations show that the model is able to create real life situations, this model can be employed for performing behavioral analysis of crowd situations with confidence.

Future developments will be using this simulation model and creating a behavior detection algorithm. Then, using that algorithm to embed behaviors in agents in crowd. 


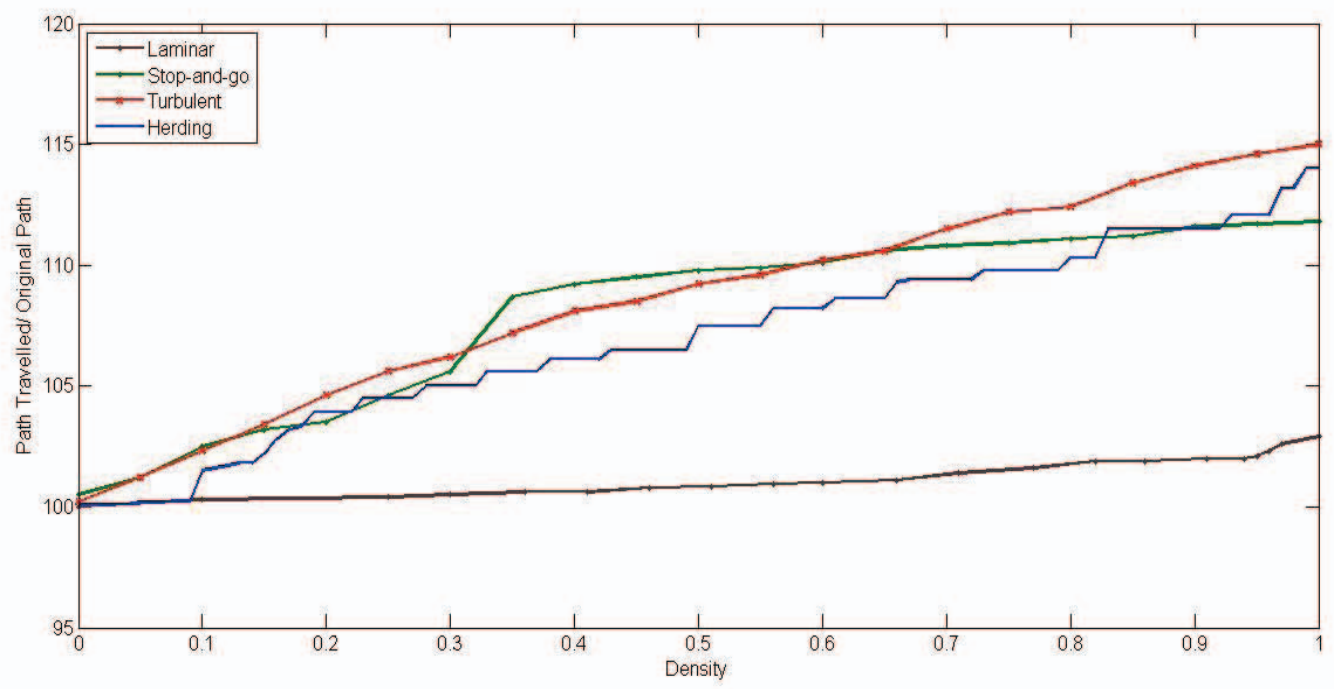

Figure 4. (a) The path travelled /original path versus density of agents [agents/in ${ }^{2}$. Different types of flows were demonstrated such as Laminar, Stopand-go waves, Turbulent and Herding behavior.

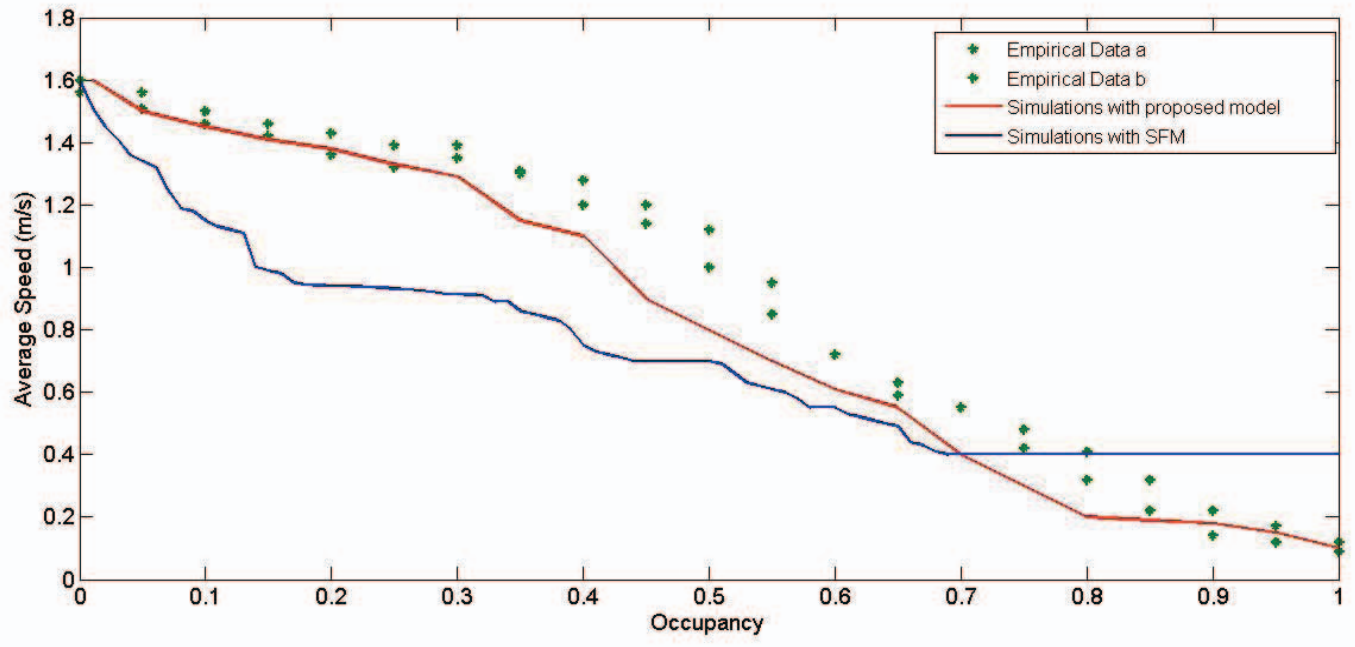

Figure 4. (b) Evaluation of RBAS model with SFM [5] and BIWI dataset [21] , using average speed versus occupancy.

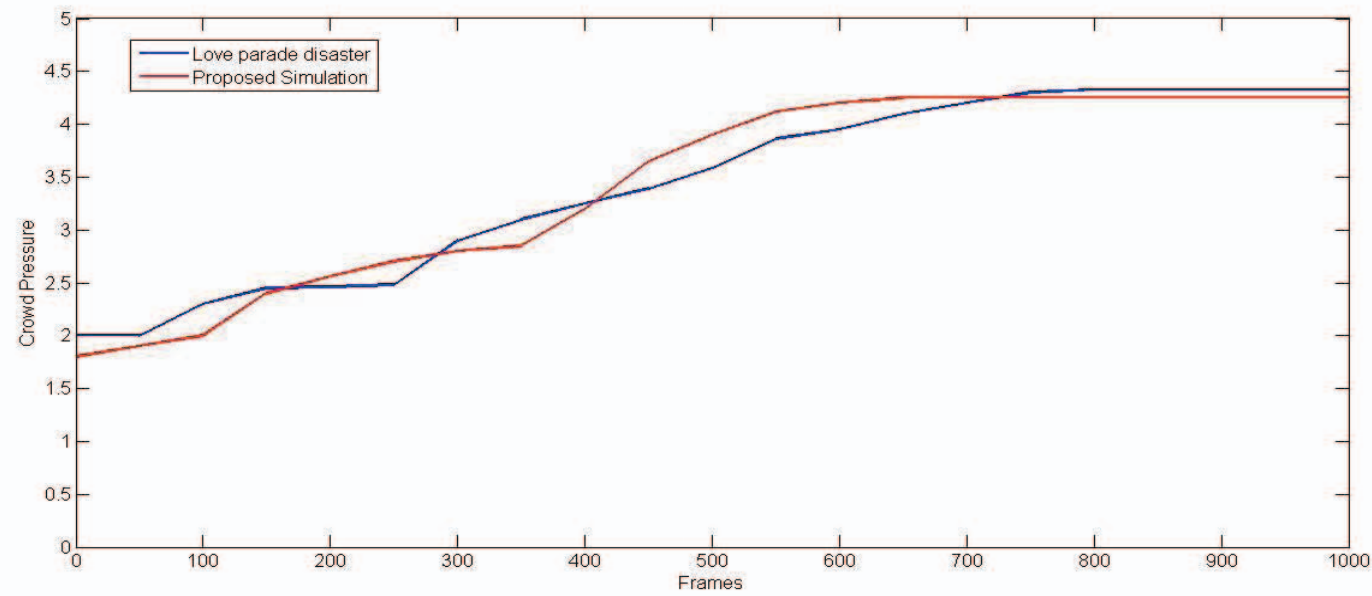

Figure 4. (c) Crowd pressure evaluation for Love Parade Disaster and using the same situation in RBAS model 


\section{ACKNOWLEDGMENT}

This work was supported in part by the Erasmus Mundus Joint Doctorate in Interactive and Cognitive Environments, which is funded by the EACEA Agency of the European Commission under EMJD ICE FPA n 2010-0012.

\section{REFERENCES}

[1] Burstedde, C., Klauck, K., Schadschneider, A., \& Zittartz, J. (2001). Simulation of pedestrian dynamics using a two-dimensional cellular automaton. Physica A: Statistical Mechanics and its Applications, 295(3), 507-525.

[2] Kirchner, A., \& Schadschneider, A. (2002). Simulation of evacuation processes using a bionics-inspired cellular automaton model for pedestrian dynamics. Physica A: Statistical Mechanics and its Applications, 312(1), 260-276.

[3] Guyot, P., \& Honiden, S. (2006). Agent-based participatory simulations: Merging multi-agent systems and role-playing games. Journal of Artificial Societies and Social Simulation, 9(4).

[4] Paris, S., Pettre, J., Donikian, S.,(2007). Pedestrian reactive navigation for crowd simulation: a predictive approach. In: Cohen-Or, D., Slavik, P. (Eds.),

[5] Karamouzas, I., Overmars, M., (2010). A velocity-based approach for simulating human collision avoidance. Lecture Notes in Computer Science 6356, 180-186

[6] Helbing, D., \& Molnar, P. (1995). Social force model for pedestrian dynamics. Physical review E, 51(5), 4282.M. Young, The Technical Writer's Handbook. Mill Valley, CA: University Science, 1989.

[7] Helbing, D., Molnar, P., Farkas, I. J., \& Bolay, K. (2001). Selforganizing pedestrian movement. Environment and planning B, 28(3), 361-384.

[8] Helbing, D., Buzna, L., Johansson, A., \& Werner, T. (2005). Selforganized pedestrian crowd dynamics: Experiments, simulations, and design solutions. Transportation science, 39(1), 1-24.

[9] Pan, X. (2006). Computational modeling of human and social behaviors for emergency egress analysis (Doctoral dissertation, Stanford University).
[10] Pressman, A. (Ed.). (2007). Architectural graphic standards. Wiley. com.

[11] Mehran, R., Oyama, A., \& Shah, M. (2009, June). Abnormal crowd behavior detection using social force model. In Computer Vision and Pattern Recognition, 2009. CVPR 2009. IEEE Conference on (pp. 935-942). IEEE.

[12] Kitazawa, K., \& Fujiyama, T. (2010). Pedestrian vision and collision avoidance behavior: Investigation of the information process space of pedestrians using an eye tracker. In Pedestrian and evacuation dynamics 2008 (pp. 95-108). Springer Berlin Heidelberg.

[13] Cutting, J. E., Vishton, P. M., \& Braren, P. A. (1995). How we avoid collisions with stationary and moving objects. Psychological review, 102(4), 627.

[14] Sakuma, T., Mukai, T., \& Kuriyama, S. (2005). Psychological model for animating crowded pedestrians. Computer Animation and Virtual Worlds, 16(3 - 4), 343-351.

[15] Moussaïd, M., Helbing, D., \& Theraulaz, G. (2011). How simple rules determine pedestrian behavior and crowd disasters. Proceedings of the National Academy of Sciences, 108(17), 6884-6888.

[16] Helbing, D., Johansson, A., \& Al-Abideen, H. Z. (2007). Dynamics of crowd disasters: An empirical study. Physical review E, 75(4), 046109.

[17] Hall, E. T., Birdwhistell, R. L., Bock, B., Bohannan, P., Diebold Jr, A. R., Durbin, M., ... \& Vayda, A. P. (1968). Proxemics [and Comments and Replies]. Current anthropology, 83-108.

[18] Guy, S. J., Chhugani, J., Kim, C., Satish, N., Lin, M., Manocha, D., \& Dubey, P. (2009, August). Clearpath: highly parallel collision avoidance for multi-agent simulation. In Proceedings of the 2009 ACM SIGGRAPH/Eurographics Symposium on Computer Animation (pp. 177-187). ACM.

[19] Guy, S. J., Chhugani, J., Curtis, S., Dubey, P., Lin, M., \& Manocha, D. (2010, July). Pledestrians: a least-effort approach to crowd simulation. In Proceedings of the 2010 ACM SIGGRAPH/Eurographics Symposium on Computer Animation (pp. 119-128). Eurographics Association.

[20] Helbing, D., Farkas, I., \& Vicsek, T. (2000). Simulating dynamical features of escape panic. Nature, 407(6803), 487-490.

[21] http://www.vision.ee.ethz.ch/datasets/

$(25 / 10 / 2013)$ 


\title{
Proceedings
}

\section{Fifth International Conference on Intelligent Systems, Modelling and Simulation}

\author{
27-29 January 2014 \\ Langkawi, Malaysia
}

Edited by

David Al-Dabass, Zaliman Sauli, and Zulkarnay Zakaria

Los Alamitos, California

Washington - Tokyo 
Copyright (C 2014 by The Institute of Electrical and Electronics Engineers, Inc.

\section{All rights reserved.}

Copyright and Reprint Permissions: Abstracting is permitted with credit to the source. Libraries may photocopy beyond the limits of US copyright law, for private use of patrons, those articles in this volume that carry a code at the bottom of the first page, provided that the per-copy fee indicated in the code is paid through the Copyright Clearance Center, 222 Rosewood Drive, Danvers, MA 01923.

Other copying, reprint, or republication requests should be addressed to: IEEE Copyrights Manager, IEEE Service Center, 445 Hoes Lane, P.O. Box 133, Piscataway, NJ 08855-1331.

The papers in this book comprise the proceedings of the meeting mentioned on the cover and title page. They reflect the authors' opinions and, in the interests of timely dissemination, are published as presented and without change. Their inclusion in this publication does not necessarily constitute endorsement by the editors, the IEEE Computer Society, or the Institute of Electrical and Electronics Engineers, Inc.

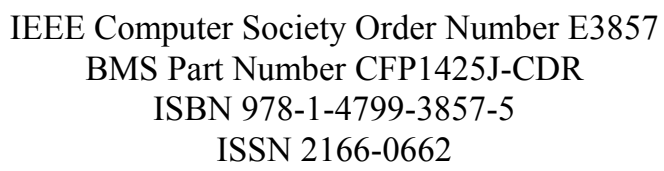

Additional copies may be ordered from:

\footnotetext{
IEEE Computer Society Customer Service Center 10662 Los Vaqueros Circle P.O. Box 3014

Los Alamitos, CA 90720-1314

Tel: + 18002726657

Fax: + 17148214641

http://computer.org/cspress csbooks@computer.org
}

IEEE Service Center 445 Hoes Lane P.O. Box 1331

Piscataway, NJ 08855-1331

Tel: + 17329810060

Fax: + 17329819667

http://shop.ieee.org/store/

customer-service@ieee.org
IEEE Computer Society Asia/Pacific Office

Watanabe Bldg., 1-4-2

Minami-Aoyama

Minato-ku, Tokyo 107-0062 JAPAN

Tel: + 81334083118

Fax: + 81334083553

tokyo.ofc@computer.org

Individual paper REPRINTS may be ordered at: <reprints@computer.org>

Editorial production by Lisa O'Conner

Art production by Mark Bartosik
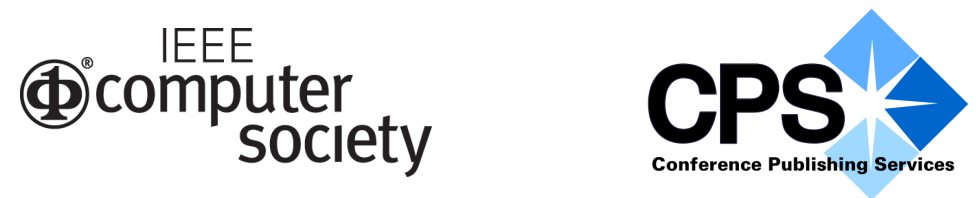

\section{IEEE Computer Society Conference Publishing Services (CPS)}

http://www.computer.org/cps 


\title{
IEEE Computer Society Technical \& Conference Activities Board
}

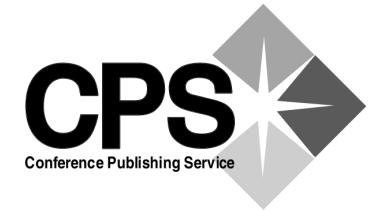

\author{
T\&C Board Vice President \\ Paul R. Croll \\ Computer Sciences Corporation
}

\author{
IEEE Computer Society Staff \\ Evan Butterfield, Director of Products and Services \\ Lynne Harris, CMP, Senior Manager, Conference Support Services \\ Alicia Stickley, Senior Manager, Publishing Operations \\ Silvia Ceballos, Manager, Conference Publishing Services \\ Patrick Kellenberger, Supervisor, Conference Publishing Services
}

IEEE Computer Society Publications

The world-renowned IEEE Computer Society publishes, promotes, and distributes a wide variety of authoritative computer science and engineering texts. These books are available from most retail outlets. Visit the CS Store at http://www.computer.org/portal/site/store/index.jsp for a list of products.

\section{IEEE Computer Society Conference Publishing Services (CPS)}

The IEEE Computer Society produces conference publications for more than 300 acclaimed international conferences each year in a variety of formats, including books, CD-ROMs, USB Drives, and on-line publications. For information about the IEEE Computer Society's Conference Publishing Services (CPS), please e-mail: cps@computer.org or telephone+1-714-821-8380. Fax +1-714-761-1784. Additional information about Conference Publishing Services (CPS) can be accessed from our web site at: http://www.computer.org/cps

Revised: 18 January 2012

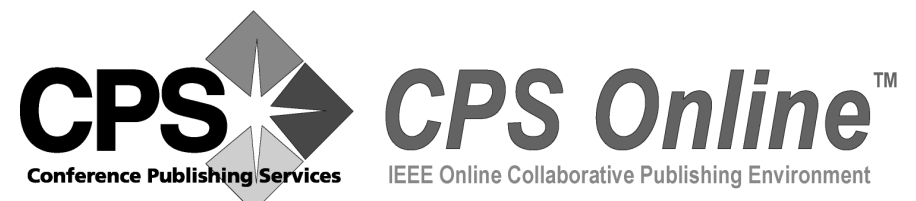

CPS Online is our innovative online collaborative conference publishing system designed to speed the delivery of price quotations and provide conferences with real-time access to all of a project's publication materials during production, including the final papers. The CPS Online workspace gives a conference the opportunity to upload files through any Web browser, check status and scheduling on their project, make changes to the Table of Contents and Front Matter, approve editorial changes and proofs, and communicate with their CPS editor through discussion forums, chat tools, commenting tools and e-mail.

The following is the URL link to the CPS Online Publishing Inquiry Form: http://www.computer.org/portal/web/cscps/quote 